\title{
Gudrun Piller
}

\section{Private Körper}

\section{Schreiben über den Körper in Selbstzeugnissen des 18. Jahrhunderts}

Erstens: Selbstzeugnisse - vor allem Autobiographien und Tagebücher - werden gemeinhin als Formen der Selbstthematisierung und damit als Instrumente der Individualisierung angesehen ${ }^{1}$. Die Entstehung und stetige Zunahme von Selbstzeugnissen gilt zudem als Charakteristikum der Zeit zwischen dem 16. und - insbesondere - dem 18. Jahrhundert.

Zweitens: Im 18. Jahrhundert entstand das Phantasma einer Identität aus der Natur des Körpers ${ }^{2}$. Ein verändertes Verhältnis des Individuums zu seinem Körper wird in der Forschung als prägend für den Übergang zur Moderne angesehen. Es entwickelte sich eine Auffassung des Körpers, der einem modernen Subjekt als Eigentum gehört und von diesem als einem bürgerlichen Individuum gelenkt wird. Erst in der Moderne wurde der Körper somit zum „Statthalter des Individuums“3, zum „Fluchtpunkt der Sinnfindung“4 oder zum „Erlebnisraum des Ich“5. Es entstand „ein neues Verständnis des Subjekts, das seine Legitimation nicht mehr aus feudaler und metaphysischer Ordnung, sondern aus der Natur des Körpers“ bezog6.

Im folgenden werden die Thematisierungen des Körpers in Selbstzeugnissen des 18. Jahrhunderts in den Blick genommen. Mit „Selbstzeugnis“ und „Körper“ stehen damit zwei Stichworte zur Debatte, die für die Frage nach Individualisierungsweisen von besonderer Bedeutung sein können, so ähnlich formuliert von Kelly und Mücke: „eighteenth-century biography is an ideal arena in which to

1 Richard van Dülmen, Die Entdeckung des Individuums. 1500-1800 (Frankfurt a. M. 1997) 85-105.

${ }^{2}$ Klaus Schneider, Natur - Körper - Kleider - Spiel. Johann Joachim Winckelmann. Studien zu Körper und Subjekt im späten 18. Jahrhundert (Würzburg 1994) 1.

3 Joachim Küchenhoff, Der Leib als Statthalter des Individuums?, in: Manfred Frank, Anselm Haverkamp (Hrsg.), Individualität (München 1988) 167-202.

${ }^{4}$ Ebd. 167.

5 Katja Patzel-Mattern, Schöne neue Körperwelt? Der menschliche Körper als Erlebnisraum des Ich, in: Körper mit Geschichte. Der menschliche Körper als Ort der Selbst- und Weltdeutung (Stuttgart 2000) 65-84.

6 Schneider, Natur - Körper - Kleider - Spiel (wie Anm. 2) 1. 
study the reciprocity between conceptions of the subject and technologies of the body"7.

Der vorliegende Text basiert auf der Analyse von fünfzig unedierten, handschriftlichen Selbstzeugnissen des 18. Jahrhunderts - darunter acht von Frauen verfaßte -, die bis vor einiger Zeit ungelesen in Archiven der Deutschschweiz lagen ${ }^{8}$. Vor dem Hintergrund der Arbeit mit diesem Quellenkorpus im Rahmen einer Dissertation soll im folgenden am Beispiel zweier ausgewählter Texte der Frage nachgegangen werden, wie sich das Schreiben über den Körper im Spannungsfeld von in der Zeit aktuellen öffentlichen Diskursen über den Körper und individuellen Interpretationen dieser Diskurse entfaltete. Das Interesse liegt dabei auch auf dem Schreiben selbst als einer Praxis, die sowohl bestimmten Normen und Vorgaben gehorcht als auch eigene Aussageabsichten verfolgt.

Die fünfzig untersuchten Texte stammen mehrheitlich aus einem städtischen Kontext, vorwiegend aus Basel und Zürich, jenen beiden Städten, die - neben Genf und Bern - in der damaligen Eidgenossenschaft über 10000 Einwohner zählten 9. Diese Orte verfügten damit über den Grad an Urbanität, der für die Partizipation an einer Kommunikationskultur entscheidend war, die ihrerseits den Nährboden für das Entstehen von Selbstzeugnissen bildete. Insbesondere in den Städten fand sich das gebildete und kommunikationswillige Publikum, das auch den Kern der schweizerischen Aufklärung ausmachte ${ }^{10}$. Unter den Verfassern der fünfzig untersuchten Selbstzeugnisse sind Fabrikanten und Kaufleute, Wissenschaftler und Professoren, Juristen und Pfarrer, aber auch ein Lehrer, ein Goldschmied, ein Apotheker, ein Zuckerbäcker oder ein Totengräber. Viele der Autoren waren als Angehörige der wirtschaftlichen Führungsschicht Mitglieder des Rats. Die acht Autorinnen gehörten eben dieser städtischen Elite an und verfaßten - oft in ihrer

7 Veronica Kelly, Dorothea von Mücke (Hrsg.), Body \& Text in the Eighteenth Century (Stanford 1994) 13.

8 Inventarisiert wurden die Texte im Rahmen des Nationalfondsprojekts „Deutschschweizerische Selbstzeugnisse (1500-1800) als Quellen der Mentalitätsgeschichte“. Das Projekt stand unter der Leitung von Prof. Dr. Kaspar von Greyerz. Bis zum Abschluß des Hauptprojekts Ende 2001 umfaßte die erstellte Datenbank 622 Texte aus der Zeit von 1500-1800. Zum Projekt vgl. Kaspar von Greyerz, Deutschschweizerische Selbstzeugnisse (1500-1800) als Quellen der Mentalitätsgeschichte. Bericht über ein Forschungsprojekt, in: Klaus Arnold, Sabine Schmolinsky, Urs Martin Zabnd (Hrsg.), Das dargestellte Ich. Studien zu Selbstzeugnissen des späteren Mittelalters und der frühen Neuzeit (Bochum 1999) 147-163; Sebastian Leutert, Gudrun Piller, Deutschschweizerische Selbstzeugnisse (1500-1800) als Quellen der Mentalitätsgeschichte. Ein Forschungsbericht, in: Schweizerische Zeitschrift für Geschichte 49 (1999) 197-221.

9 Rudolf Braun, Das ausgehende Ancien Régime in der Schweiz. Aufriß einer Sozial- und Wirtschaftsgeschichte des 18. Jahrhunderts (Göttingen, Zürich 1984).

10 In der jüngeren Aufklärungsgeschichte wird Aufklärung im wesentlichen als ein sozialer Prozeß gesehen, der mit der Intensivierung der Kommunikationsprozesse in der Gesellschaft des 18. Jahrhunderts zusammenhängt. Zur Aufklärung als Kommunikation vgl. Hans Erich Bödeker, Aufklärung als Kommunikationsprozeß, in: Rudolf Vierhaus (Hrsg.), Aufklärung als Prozeß (Hamburg 1987) 89-111. Zur Schweiz und zu einzelnen Städten als Orte der Aufklärung vgl. Brigitte Schnegg, „Die zweyte Seite auf dem Blatte der Menschheit“. Geschlechterdiskurse und Geschlechterverhältnisse in der Schweizer Aufklärung (Bern 1999) 10-65. 
Funktion als Erzieherinnen - ein Selbstzeugnis für ihre Kinder oder übernahmen die (Weiter-)Führung eines Familienbuches. Formal handelt es sich bei den Texten um Autobiographien, längere Lebensläufe, Hausbücher, Familienbücher, Tagebücher, jährliche Notizen, Notizbücher in Jahreskalendern, Generationen übergreifende Familienbücher oder Selbstzeugnisse mit Schwerpunktthemen, wie Erziehung oder Ehe, wobei die Formen in vielfacher Weise ineinandergreifen können.

Ein verbindendes Merkmal ist, daß die Texte keinem administrativ-institutionellen Kontext entstammen, sondern im privaten Rahmen entstanden sind. Mit „privatem Rahmen“ ist allerdings nicht die für das späte 18. Jahrhundert beschriebene Dissoziation von Privatheit und Öffentlichkeit und die damit assoziierte Trennung der Lebensbereiche von Frauen und Männern gemeint ${ }^{11}$. In Selbstzeugnissen berichten Frauen und Männer über sogenannt „Privates“ wie Geburten, Krankheiten oder Todesfälle ebenso wie über öffentlichkeitsrelevante Themen wie Patenschaften, Geschäftliches und Politisches. Es präsentiert sich darin ein Bild des familiären Lebens, in dem Privates und Öffentliches vielfältig zusammenlaufen. Die bürgerlichen Häuser, aus denen die Texte größtenteils stammen, sind keine Refugien der Privatheit, sondern Orte der Kommunikation und der familiären Geselligkeit ${ }^{12}$. Dennoch sind die Texte in der „heimlichen Stube“ ${ }^{13}$, am häuslichen Tisch entstanden und sind zumeist - explizit oder implizit - an die Familie adressiert.

Daß sich unter den Autoren auch ein Grundschullehrer, ein Apotheker, ein Zuckerbäcker, ein Goldschmied oder ein Totengräber finden, macht deutlich, daß die Verfasser nicht ausschließlich aus dem Zentrum der städtischen Elite stammen ${ }^{14}$. In den Worten Andrew Wears ist die Geschichte, die auf der Basis dieser Quellen geschrieben wird, somit „not 'history from below'“, aber „history from the middle, the history of literate but often unimportant people“ 15 .

Dies trifft mehr oder weniger stark auch auf die beiden Autoren zu, deren Texte für die folgenden Überlegungen als Beispiele herangezogen werden: den Zürcher

11 Kritisch dazu vgl. Karin Hausen, Öffentlichkeit und Privatheit. Gesellschaftspolitische Konstruktion und die Geschichte der Geschlechterbeziehungen, in: Karin Hausen, Heide Wunder (Hrsg.), Frauengeschichte - Geschlechtergeschichte (Frankfurt a. M., New York 1992) 81-88.

12 Vgl. Anne-Charlott Trepp, Sanfte Männlichkeit und selbständige Weiblichkeit. Frauen und Männer im Hamburger Bürgertum zwischen 1770 und 1840 (Göttingen 1996).

13 „Geheim“ und „heimlich“ waren die im 16./17. Jahrhundert für die Übersetzung von „privatus“ oft benutzten deutschen Wörter. Die Beschreibung der „heimliche[n] Stube, in der man daheim, in der einem wohl ist", stammt aus Johann Heinrich Campes, Wörterbuch der Deutschen Sprache von 1808. Vgl. Hausen, Öffentlichkeit und Privatheit (wie Anm. 11) 84.

${ }^{14}$ Der bürgerliche Anteil der Stadtbewohner betrug in Zürich rund 60\% (1780) und in Basel 55\% (1779). Auch dieser bürgerliche Teil bildete jedoch keinesfalls eine Einheit, sondern kannte Privilegierte und Minderprivilegierte. Sowohl in politischer wie in sozioökonomischer Hinsicht waren die Approbationschancen unterschiedlich verteilt. Vgl. Braun, Das ausgehende Ancien Régime in der Schweiz (wie Anm. 9) 161f.

15 Andrew Wear, Interfaces: Perceptions of Health and Illness in Early Modern England, in: Roy Porter, Andrew Wear (Hrsg.), Problems and Methods in the History of Medicine (London, New York, Sydney 1987) 230-255, 231. 
Kaufmann und Bürgermeister Johann Caspar Escher (1678-1762) und den Zürcher Totengräber Hartmann Wirz (1727-1795). Johann Caspar Escher verfaßte in der Zeit zwischen 1745 und 1750 eine in vier Teile gegliederte Autobiographie, deren ersten Teil er unter dem Titel „Mein Leibs und gesundheits-beschaffenheit“ ganz seiner physischen Entwicklung und den körperlich relevanten Stationen seines Lebens widmete ${ }^{16}$. Nicht über seinen eigenen Körper, sondern über den Körper, die Krankheit und den Tod eines fünfjährigen Kindes im Jahr 1764 schrieb der Totengräber Hartmann Wirz und schuf damit einen Text, der aus verschiedenen Gründen sehr bemerkenswert ist ${ }^{17}$.

Johann Caspar Escher kann nicht als unbedeutend bezeichnet werden. Immerhin gilt er als einer der einsichtigsten und aufgeklärtesten Staatsmänner der alten Schweiz ${ }^{18}$. Mit seiner in vier Teile gegliederten Autobiographie verfaßte er einen Text, der ihn als exemplarischen, aber zugleich eigenständigen Angehörigen des städtischen Bürgertums ausweist. Den ersten Teil widmete er unter dem Titel „Mein Leibs und gesundheits-beschaffenheit“ seiner physischen Entwicklung und den körperlich relevanten Stationen seines Lebens ${ }^{19}$.

Escher läßt in keiner Zeile Zweifel daran aufkommen, daß mit dieser Geschichte das Leben eines erfolgreichen Mannes erzählt wird. Wichtige Voraussetzung einer solchen Erfolgsgeschichte ist ein gesunder Körper - dies eine mögliche Erklärung dafür, daß Escher das Kapitel über Leib und Gesundheit an den Anfang stellt. Gesunde Körper gehörten zum Kapital des sich im Laufe des 18. Jahrhunderts etablierenden Bürgertums, wobei der bürgerliche Normendiskurs Gesundheit sowohl als Voraussetzung wie als Folge eines rechtschaffenen und geordneten Lebens behandelte. Der Körper war ein optimierbares Potential und entschied über die zur Verfügung stehenden physischen und psychischen Kapazitäten. Die Erhaltung der Gesundheit wurde damit zur grundlegenden und permanenten Lebensaufgabe, nicht nur im Sinne eines privaten Wunsches, sondern als Pflicht gegenüber der Allgemeinheit ${ }^{20}$. Das erste Kapitel der Autobiographie ist Eschers Darlegung seiner Erfüllung dieser Aufgabe.

16 Johann Caspar Escher d.J. (1678-1762), o. T. Ms. Handschriftenabteilung der Zentralbibliothek Zürich, FA von Wyss III 116 und III116a.

17 Hartmann Wirz (1727-1795), o. T. Ms. Handschriftenabteilung der Zentralbibliothek Zürich, FA Wirz 137.2.

$18 \mathrm{Zu}$ Johann Caspar Escher vgl. Michael Kempe, Thomas Maissen, Die Collegia der Insulaner, Vertraulichen und Wohlgesinnten in Zürich 1679-1709. Die ersten deutschsprachigen Aufklärungsgesellschaften zwischen Naturwissenschaften, Bibelkritik, Geschichte und Politik (Zürich 2002) 128-134.

19 Im vierten und längsten Teil berichtet er über sein öffentliches Leben und seine Amtsgeschäfte, im dritten Teil über seine Ausbildung und seine Reisen und im zweiten über die religiösen Aspekte seines Lebens. Der ganze Text umfaßt 370 Seiten. Auf den ersten Teil entfallen 41 Seiten.

20 Zum aufklärerischen Gesundheitsdiskurs vgl. z. B. Christian Barthel, Medizinische Polizey und medizinische Aufklärung. Aspekte des öffentlichen Gesundheitsdiskurses im 18. Jahrhundert (Frankfurt a. M., New York 1989); Peter Borscheid, Für ein langes Leben. $\mathrm{Zu}$ den Bemühungen um eine höhere Lebenserwartung im Zeitalter des Absolutismus, in: 
Sein Körperwissen bezieht Escher aus verschiedenen Quellen. Zum einen ist er ein Leser. Seine physische Selbsterkundung und diätetische Auseinandersetzung ist stets von Lektüre begleitet. Er konsultiert zeitgenössische Autoren ebenso wie Francis Bacon und Hippokrates, in dessen Aphorismen er seinen eigenen Zustand so deutlich dargestellt findet, wie wenn er ihn selbst beschrieben hätte. Ganz maßgeblich aber ist Eschers Körperwahrnehmung gestützt durch den Kontakt und das Gespräch mit den Personen, die ihn umgeben. Dies können Ärzte sein, aber auch nicht-professionelle Personen, wie etwa seine Haushälterin:

„unterschiedliche Remedica von Medicis haben nit wollen helfen, da hat mir unsere haushalterin die Jgfr Elisabeth Schinz erzählet, als sie vor einigen Jahren mit gleichen Schmerzen geplagt worden, hab jemand ihr gerathen .... ${ }^{21}$.

Eschers daraufhin erfolgreiche Anwendung des empfohlenen Mittels verweist auf die Bedeutung von mündlich tradiertem Erfahrungswissen und zeigt, daß in der Verhandlung von Körperwissen die Schichtgrenzen durchlässig waren ${ }^{22}$. Es kann hinsichtlich ärztlicher und sogenannter Volksmedizin nicht von zwei strikt voneinander getrennten Sphären ausgegangen werden ${ }^{23}$.

Eschers ständige Auseinandersetzung mit Körperwissen ist begründbar. Seine Aufgabe, den Körper zu erhalten, ist nämlich keine einfache, ist er doch „von zarter Leibesbeschaffenheit“ ${ }^{24}$. Ein Arzt attestiert ihm zu diesem zarten Leib einen sehr lebendigen Geist, also einen für das 18. Jahrhundert typischen Gelehrtenkörper $^{25}$. Ein Unfall in der Kindheit hat lebenslang einen schwächeren Atem zur Folge, seiner Pockenerkrankung wäre er beinahe erlegen, und während seiner Studienreisen macht er mehrere Krankheiten durch. Stets betont er jedoch, kaum auf die Hilfe von Ärzten zurückgegriffen zu haben. Rückblickend beurteilt er sich in Kindheit und Jugend als „ziemlich gesund“ 26 .

Außerdem bezeichnet er seine Erkrankungen nicht als „Krankheiten“, sondern als „Gesundheitsanstösse“. Während die Begriffe „Krankheit“ und der bei Escher und im allgemeinen Gebrauch synonym dazu verwendete Begriff „Zufall“ in die Terminologie des 18. Jahrhunderts gehören, handelt es sich bei „Gesundheits-

Nils-Arvid Bringéus u. a. (Hrsg.), Wandel der Volkskultur in Europa, Bd.2 (Münster 1988) 697-708; Mary Lindemann, „Aufklärung“ and the Health of the People. „Volksschriften“ and Medical Advice in Braunschweig-Wolfenbüttel, 1756-1803, in: Rudolf Vierhaus (Hrsg.), Das Volk als Objekt obrigkeitlichen Handelns (Tübingen 1992) 101-120.

21 Escher, o. T. 11.

22 Zur mündlichen Weitergabe von Wissen vgl. Regina Schulte, Bevor das Gerede zum Tratsch wird, in: Karin Hausen, Heide Wunder (Hrsg.), Frauengeschichte - Geschlechtergeschichte (Frankfurt a. M., New York 1992) 67-73.

${ }^{23} \mathrm{Vgl}$. Eberhard Wolff, „Volksmedizin“ als historisches Konstrukt. Laienvorstellungen über die Ursachen der Pockenkrankheit im frühen 19. Jahrhundert und deren Verhältnis zu Erklärungsweisen der akademischen Medizin, in: Österreichische Zeitschrift für Geschichtswissenschaften 7 (1996) 405-430.

24 Escher, o. T. 2.

25 Gustav Adolf Wehrli, Die Berufskrankheiten des Gelehrtenstandes im 18. Jahrhundert, in: Praxis. Schweizerische Rundschau für Medizin XVIII (1929) 44-46, [1]-23.

26 Escher, o. T. 2. 
anstoss“ um eine individuelle Wortverwendung, eine Wortschöpfung Eschers. Mit dem Wort „Gesundheitsanstoss“ schafft sich der stark auf Darstellung seiner Gesundheit bedachte Escher die Möglichkeit, über seine körperlichen Insuffizienzen zu berichten, ohne sie als „Krankheiten“ deklarieren zu müssen. Wenn Escher zudem erläutert, wie er im Kontext seiner „Gesundheitsanstösse“ mit einfachen Heilmitteln Erfahrungen sammelt, die er im Verlauf seines weiteren Lebens „oft glücklich gebraucht“27, erhält der „Gesundheitsanstoss“ eine geradezu positive Komponente: Situationen körperlicher Schwäche oder Bedrohung werden zum Umschlagplatz für Deutungsmuster und medizinisches Wissen. Der „Gesundheitsanstoss“ ist damit keineswegs eine Krankheit, sondern vielmehr ein Anstoß zu langem Gesundsein.

Eschers bisweilen geradezu obsessiv wirkendes Postulieren steter Gesundheit, das Negieren von Krankheit oder der Inanspruchnahme medizinischer Dienstleistungen reflektieren ein ambivalentes Verhältnis zur Medizin, zu ihren Methoden und ihren Vertretern. Gerd Göckenjan beschrieb diese Skepsis als eine Art Selbstregulationsdoktrin, die insbesondere im liberalen Bürgertum anzutreffen war. Sie stütze sich auf die prinzipielle Selbstverantwortlichkeit des Individuums, das durch eine mäßige Lebensordnung Krankheit verhindern sollte ${ }^{28}$. Friedrich Hoffmann schrieb 1715, daß ein jeder „sein eigener Arzt sein und an sich wahrnehmen müsse, worauf er sich übel oder wohl befinde “29, und auch 1798 sollte medizinische Aufklärung bedeuten, „über sein physisches Wohl selbst zu denken“30.

Escher verfolgt diesen Weg durch eine intensive Auseinandersetzung mit der diätetischen Lebensweise, die er im Sinne einer Empirie am eigenen Leib betreibt. Unter Diätetik wurde seit der Antike die ärztliche Kunst verstanden, alle äußeren Gegebenheiten, die auf das Befinden des Menschen einwirkten, unter Berücksichtigung der individuellen Konstitution ins rechte Maß zu bringen ${ }^{31}$. Und auch am Ende der langen medizinischen Tradition der Diätetik, in Johann Peter Franks zwischen 1779 und 1819 erschienenem sechsbändigen „System einer vollständigen medizinischen Polizey“ galt noch immer die Diätetik als zentrale Aufgabe eines jeden einzelnen und war Ausgangspunkt jeder Form von Prävention und Thera$\mathrm{pie}^{32}$. Eine diätetische Lebensweise bedeutete somit nicht das Befolgen eines stren-

27 Escher, o. T. 4.

28 Gerd Göckenjan, Kurieren und Staat machen. Gesundheit und Medizin in der bürgerlichen Welt (Frankfurt a. M. 1985) 85.

${ }^{29}$ Friedrich Hoffmann, Gründliche Anweisung wie ein Mensch vor dem frühzeitigen Tod und allerhand Arten Kranckheiten durch ordentliche Lebens-Art sich verwahren könne, 3 Theile (Halle 1715-1717) zitiert nach: Göckenjan, Kurieren und Staat machen (wie Anm. 28) 85.

30 Johann Karl Osterbausen, Über medizinische Aufklärung (Zürich 1798) zitiert nach: Göckenjan, Kurieren und Staat machen (wie Anm. 28) 85.

31 Ursula Weisser, Hippokrates/Galen, in: Dietrich v. Engelhardt, Fritz Hartmann (Hrsg.), Klassiker der Medizin, Bd. 1 (München 1991) 12-29, 25.

32 Johann Peter Frank, System einer vollständigen medicinischen Polizey, 6 Bde. (Mannheim [Bd.1-4], Tübingen [Bd. 5], Wien [Bd.6] 1779-1819). Dazu vgl. Eduard Seidler, Johann Peter Frank (1745-1821), in: Dietrich v. Engelhardt, Fritz Hartmann (Hrsg.), Klassiker der Medizin, Bd. 1 (München 1991) 291-308, 307. 
gen Vorschriftenkatalogs. Vielmehr paßt Escher seine diätetischen Lebensgewohnheiten immer wieder den sich wandelnden Lebensumständen und den altersbedingten Veränderungen an und entspricht mit diesem Vorgehen der im 18. Jahrhundert gängigen Diätetik-Auffassung 33 . Die Möglichkeit der individuellen und selbst verwalteten Anwendung der Diätetik war zweifellos ein Vorteil im Kontext der aufgeklärten Medizin des 18. Jahrhunderts sowie ein Grund für ihre bis ins 19. Jahrhundert wirkende Beharrungskraft.

„[M]it dieser Diät und diesen Observationen hab’ durch Gottes Güte eine zarte, schwache Gesundheit jetzt bald bis an das 69ste Jahr erhalten, ohne daß ich außer den Kinderblattern in dieser so langen Zeit jemahl mehr als 3 oder 4 Tag Krankheit halber müssen im Bett liegen, oder sonst mein Geschäft versäumen; deßwegen ich es der Würde geachtet meinen lieben Kindern davon Bericht zu geben, um Ihnen zu erweisen, daß ich nit par caprice oder menage die Medicos, Arzneien, Badenkuren, Sauerbrunnen u.s.w. gesuchen; und sie aufzumuntern, daß sie vornehmlich selber auf ihr Gesundheit Achtung geben."34

In dieser von Escher quasi als Quintessenz formulierten Textstelle sind die Elemente enthalten, die Johann Caspar Escher als Einzelfall für die Analyse interessant machen und ihn als exemplarischen und gleichzeitig eigenständigen Angehörigen des städtischen Bürgertums ausweisen. Eschers Fokus richtet sich auf die Gesundheit, die in verschiedenen Schattierungen ständig präsent ist: als Gabe Gottes, als tägliche Aufgabe, als Bedingung für Produktivität und vor allem als Verdienst und Ergebnis steter eigener Bemühungen. Tatsächlich wird Escher 84 Jahre alt und überlebt alle seine vierzehn Kinder, an die er seine Erkenntnisse weitergeben will ${ }^{35}$.

Escher liefert eine Sicht auf die Geschichte seines Körpers, in der es ihm gelingt, einen durch die Polarität von starkem Geist und schwachen Körper permanent gefährdeten Leib durch eigenen Einsatz bis ins Alter gesund, das heißt leistungs- und arbeitsfähig zu erhalten. In verschiedenen Funktionen wird der Körper als konstitutiver Bestandteil von Eschers Selbstverständnis und mithin für die Konstruktion von Identität sichtbar. Eschers schwacher aber gesunder Körper ist - in Kombination mit dem darin sitzenden starken Geist - die Voraussetzung für ein störungsfreies Arbeitsleben, und der Körper ist gleichzeitig das Anschauungsobjekt, an dem Escher den Erfolg von seriöser Arbeit - in diesem Fall Gesundheitsvorsorge und Diätetik - für seine Nachkommen wirksam zu demonstrieren weiß.

Die Geschichte seiner „Leibs und gesundheits-beschaffenheit“, die Escher zwischen 1745 und 1750 verfaßte, liest sich bisweilen wie eine autobiographische Umsetzung des aufklärerischen Gesundheitsdiskurses, wie ihn Gerd Göckenjan und andere in der Analyse der Schriften von Tissot, Frank, Senfft, Faust, Hufeland, Zimmermann u.a. herausgearbeitet hat. Gesundheit wird darin als Ergebnis von Vernunft, Arbeit, Mäßigkeit und Disziplin beschrieben ${ }^{36}$. Vor diesem Hintergrund

33 Vgl. dazu Göckenjan, Kurieren und Staat machen (wie Anm. 28) 84.

34 Escher, o. T. 16.

35 Kempe, Maissen, Die Collegia der Insulaner (wie Anm. 18) 133.

36 Göckenjan, Kurieren und Staat machen (wie Anm. 28) 90. 
reflektiert auch Eschers Skepsis gegenüber Ärzten und medizinischen Eingriffen die Doktrin der Selbstregulation in Belangen der Gesundheit. Mit seiner selbst verwalteten Gesundheitspflege scheint Escher die Paradigmen und normativen Zielsetzungen der medizinischen Aufklärung allerdings geradezu vorwegzunehmen. Denn auf dem Höhepunkt angelangt war der aufklärerische Gesundheitsdiskurs erst in der zweiten Hälfte des 18. Jahrhunderts ${ }^{37}$. Eschers Text ist somit ein Hinweis darauf, daß Autoren von Selbstzeugnissen nicht einfach auf dominante Diskurse aufspringen und darauf Bezug nehmen, sondern selber aktiv an deren Produktion mitarbeiten.

Häufiger als über den eigenen Körper wird in Selbstzeugnissen des 18. Jahrhunderts über den Körper von Familienangehörigen geschrieben. Außerdem wird der Körper in den meisten Fällen erst dann zum Thema, wenn er krank, also bedroht ist. Der typische Fall ist, daß nach dem Tod eines nahen Angehörigen die Krankengeschichte kurz resümiert wird. Aus einem Korpus von fünfzig Texten ist damit Johann Caspar Eschers Autobiographie als besonderer Text herausragend, da darin die Geschichte des eigenen Körpers als wichtiger Bestandteil des umfassenden Selbstverständnisses erzählt wird.

Der zweite Text, der kurz vorgestellt werden soll, ist das Selbstzeugnis des Zürcher Totengräbers Hartmann Wirz. Auch dieser Text zeigt interessante Bewegungen zwischen öffentlichen Diskursen der Zeit und der eigenständigen Umsetzung dieser Diskurse. In Wirz' Text drängt sich allerdings stärker die Frage nach dem Verhältnis von traditionellen Normen des Selbstzeugnisschreibens und dem individuellen Umgang mit diesen Normen resp. dem Bruch damit in den Vordergrund.

Der Text von Hartmann Wirz aus den Jahren 1754-1764 ist weder eine Autobiographie noch ein Tagebuch, gehört also nicht zu jener Sorte von Selbstzeugnissen, der die größte Bedeutung für den Vorgang der Individualisierung zugesprochen wird. Mit Wirz' Text liegt eine andere für das 18. Jahrhundert nach wie vor wichtige Form von Selbstzeugnis vor: das Haus- oder Familienbuch zur Aufzeichnung von Familienereignissen, insbesondere von Geburten, Taufen und Todesfällen. In Familienbüchern wird die enge Verflechtung von Individuum und Familie manifest: Die Familie bildete Ausgangs- und Zielpunkt frühneuzeitlicher Selbstverortung. In der Tradition der Haus- und Familienbücher, die sich vom 16./17. bis ins 18. Jahrhundert hinein fortsetzte, zeigt sich diese Art von auf die Familie bezogener Selbstthematisierung ${ }^{38}$.

Die übliche Form, wie Ereignisse in den Familienbüchern festgehalten werden, sind eher kurze, bisweilen geradezu standardisierte Notizen. Dies gilt nicht nur für Geburten, Taufen oder Hochzeiten, sondern auch für Todesfälle, ebenso für Todesfälle von Kindern. Und gerade der Tod von Kindern ist in Selbstzeugnissen,

37 Die bei Göckenjan untersuchten Schriften stammen mehrheitlich aus der Zeit nach 1750. Vgl. auch Michel Foucault, Der Wille zum Wissen (Frankfurt a. M. 1983) 161ff.

38 Vgl. Natalie Zemon Davis, Die Geister der Verstorbenen, Verwandtschaftsgrade und die Sorge um die Nachkommen. Veränderungen des Familienlebens in der frühen Neuzeit, in: dies., Frauen und Gesellschaft am Beginn der Neuzeit (Frankfurt a. M. 1989) 19-51. 
vor allem in Familienbüchern und Familienaufzeichnungen, ein häufig berichtetes Ereignis.

Die Baslerin Ursula Merian-Burckhardt (1752-1833) beispielsweise registrierte in ihren Familiennotizen den Tod von fünf ihrer neun Kinder, alle in identischer Weise ${ }^{39}$ :

„1782 d 2. Aprill ist mein geliebtes Kind Salome in dem Herrn Sanfft und Seelig entschlaffen nachmittag zwischen 2 und $3 \mathrm{uhr}$; und ist d 4. zu St. todor begraben worden; der liebe Gott wolle ihme Eine freüliche auferstendnuß verleichen und uns allen eine Seilige nachfard in Christo Jesu Amen; es ist 5 Jahr 5 Wochen 3 Tag alt worden." 40

Ohne Unterschied, ob es sich dabei um ein einjähriges oder ein fünfjähriges Kind handelte, notiert die Mutter den Tod mit Angabe des Sterbedatums, der Sterbestunde, des Begräbnisorts und des genauen Alter des Kindes sowie mit dem Hinweis, daß das Kind „sanft und selig entschlafen“ sei. Nie hingegen gibt Merian-Burckhardt an, ob das Kind krank war oder welche Todesursache angenommen wird.

Es ist üblich, daß in Zusammenhang mit derlei kurzen Todesnotizen auf die hohe Säuglings- und Kindersterblichkeit verwiesen wird. Mit rund 25\% vor dem Erreichen des 1. Altersjahres und rund 50\% vor dem Erreichen des 10. Altersjahres blieben die Sterblichkeitsraten bis weit ins 19. Jahrhundert hinein unverändert hoch $^{41}$. Dies, obwohl im 18. Jahrhundert dem Kinderkörper vermehrte Aufmerksamkeit zukam und in dieser Epoche der Grundstein für die Pädiatrie gelegt wur$\mathrm{de}^{42}$. Zunehmend wurden auch medizinische Dissertationen zu pädiatrischen Themen verfaßt ${ }^{43}$. Dabei wurden zahlreiche Kinderkrankheiten Gegenstand genauer Beschreibung ${ }^{44}$. Insbesondere wurden gegen die Pocken wirksamere Vorbeu-

39 Die fünf während der Berichtzeit verstorbenen Kinder waren Salome (1777-1782), Rudolf (1778-1779), Maria Magdalena (1780-1783), Anna Catharina (1783-1788) und Remigius (1786-1792). Sie starben alle vor dem Erreichen des sechsten Altersjahres.

40 Ursula Merian-Burckhardt (1752-1833), o. T., Ms. Staatsarchiv Basel-Stadt, ohne Paginierung, PA 101 A 2,2, 6.

41 In einigen Fällen liegen die errechneten Werte im 19. Jahrhundert sogar höher als im 18. Jahrhundert. Vgl. z. B. Iris Ritzmann, Kinderkrankheiten und Kindersterblichkeit, in: Paul Hugger (Hrsg.), Kind sein in der Schweiz (Zürich 1998) 301-317, 312; Markus Mattmüller, Medizingeschichte und allgemeine Historie - Dialog und Zusammenarbeit auf dem Gebiet der modernen Sozialgeschichte, in: Gesnerus 37 (1980) 1/2, 62-72, 69; Richard van Dülmen, Kultur und Alltag in der Frühen Neuzeit, Bd.1 (München 1990) 88; Jean-Louis Flandrin, Familien. Soziologie - Ökonomie - Sexualität (Frankfurt a. M., Berlin, Wien 1978) 320; Arthur E. Imhof, Einführung in die Historische Demographie (München 1977) 67; Albrecht Peiper (Hrsg.), Chronik der Kinderheilkunde (Leipzig 51992) 402; Max Baer, Medizinisch statistische Ergebnisse aus zürcher. Kirchenbüchern des 17. und 18. Jahrhunderts (Zürich 1926) 11.

42 Johannes Oehme, Medizin der Aufklärung unter besonderer Berücksichtigung von Kinderkrankheiten (Dokumenta Paediatrica, Lübeck 1986); Erwin H. Ackerknecht, Geschichte der Medizin (Stuttgart 1992) 100; Heinrich Schipperges (Hrsg.), Geschichte der Medizin in Schlaglichtern (Mannheim 1990) 228.

43 Oebme, Medizin der Aufklärung (wie Anm. 42) 31-62.

44 Jean-Noël Biraben, Arzt und Kind im 18. Jahrhundert. Bemerkungen zur Pädiatrie des 18. Jahrhunderts, in: Arthur E. Imbof (Hrsg.), Biologie des Menschen in der Geschichte. 
gungsmaßnahmen entwickelt, und die Durchsetzung der Kuhpockenimpfung durch Eduard Jenner im Jahr 1796 gilt in der Medizingeschichtsschreibung als eines der wichtigsten medizinischen Ereignisse des 18. Jahrhunderts überhaupt ${ }^{45}$. Auch Michel Foucault hob im Rahmen seiner Überlegungen zum Medikalisierungsprozeß und zur Politik der Gesundheit im 18. Jahrhundert die „Privilegierung der Kindheit" besonders hervor ${ }^{46}$.

Tatsächlich äußerte sich dieses vermehrte Interesse am Kinderkörper im 18. Jahrhundert jedoch noch nicht in einer Abnahme der Säuglings- und Kindersterblichkeit. Auch im besten bürgerlichen Hause der Ursula Merian-Burckhardt werden fünf der neun Kinder vor dem Erreichen des sechsten Altersjahrs zu Grabe getragen.

Noch immer genannt, aber kaum mehr positiv gewertet, werden Erklärungen wie jene Edward Shorters, der in den 1970er Jahren mit seiner Studie „The Making of the Modern Family“ nachhaltig die These von der Gleichgültigkeit der traditionalen Gesellschaft gegenüber ihren Kindern prägte ${ }^{47}$. Shorter betonte, daß der für ihn offensichtliche Mangel an elterlicher Liebe und die emotionale Kälte nicht bloß eine Folge der hohen Kindersterblichkeit gewesen seien, sondern „gerade die unzureichende Zuneigung und Pflege an der hohen Sterblichkeit schuld war." 48 Unterdessen ist diese Vorstellung - nicht zuletzt durch die Arbeit mit Selbstzeugnissen - vielfach widerlegt ${ }^{49}$.

Hartmann Wirz begann mit der Aufzeichnung familiärer Ereignisse im Jahr seiner Eheschließung 1754. Er verfolgte seine Schreibtätigkeit bis nach dem Tod der

Beiträge zur Sozialgeschichte der Neuzeit aus Frankreich und Skandinavien (Stuttgart-Bad Cannstatt 1978) 261-273.

45 Wolfgang U. Eckart, Geschichte der Medizin (Berlin, Heidelberg, New York 1994) 203. Beschrieben wurden die Pocken bereits in den sechziger Jahren des 17. Jahrhunderts durch den englischen Arzt Thomas Sydenham (1624-1689). Im 18. Jahrhundert waren sie die häufigste Todesursache von Kindern. Die breitenwirksame Durchsetzung der Pockenimpfung reichte dann allerdings weit ins 19. Jahrhundert hinein. Zu den Problemen bei der Durchsetzung der Pockenschutzimpfung im frühen 19. Jahrhundert vgl. Wolff, „Volksmedizin“ als historisches Konstrukt (wie Anm. 23).

46 Michel Foucault, Die Politik der Gesundheit im 18. Jahrhundert, in: Österreichische Zeitschrift für Geschichtswissenschaften 7 (1996) 311-326.

47 Edward Shorter, The Making of the Modern Family (New York 1975). Zur angeblichen Gleichgültigkeit gegenüber den Kindern in früherer Zeit, die sich besonders darin gezeigt haben soll, wie gleichmütig der Tod eines Kindes registriert wurde vgl. auch Philippe Ariès, Geschichte der Kindheit (München 1975) 99 (L’enfant et la vie familiale sous l'ancien régime, Paris 1960). Auch Beutelspacher zweifelt an der Beziehung der Eltern zu den Kindern mit der Begründung, „bei der hohen Kindersterblichkeit wären die Eltern sonst aus dem Trauern nicht mehr herausgekommen“. Vgl. Martin Beutelspacher, Kultivierung bei lebendigem Leib. Alltägliche Körpererfahrung in der Aufklärung (Weingarten 1986) 53.

48 Edward Shorter, Die Geburt der modernen Familie (Reinbek bei Hamburg 1977) 234.

49 Linda Pollock, Forgotten Children. Parent-Child Relations from 1500 to 1900 (Cambridge 1983); Anne-Charlott Trepp, Sanfte Männlichkeit (wie Anm. 12) 338-342; Olwen Hufton, Arbeit und Familie, in: Geschichte der Frauen, Bd.3, hrsg. von Arlette Farge und Natalie Zemon Davis (Frankfurt a. M., New York 1994) 27-59; Paul Hugger, Meister Tod. Zur Kulturgeschichte des Sterbens in der Schweiz und in Lichtenstein (Zürich 2002) 162-188. 
Tochter Anna Maria im Jahr 1764. Die zwei am ausführlichsten berichteten Ereignisse sind die Krankengeschichten seiner Kinder Hartmann und Anna Maria. Hartmann starb am 8. Juni 1764 im Alter von neun Monaten, Anna Maria eine Woche später im Alter von fünf Jahren. Mit 36 Seiten Umfang nimmt Anna Marias Lebens-, Krankheits- und Sterbegeschichte 60\% des Textes in Anspruch.

Mit der Ausführlichkeit des Berichts und der emotionalen Intensität der Beschreibung weicht Wirz außerordentlich stark von der in Haus- und Familienbüchern üblichen Form, Todesfälle von Kindern zu registrieren, ab. Warum es zu solchen Abweichungen von der Norm kommt, läßt sich letztlich oft - auch in diesem Fall - nicht erklären.

Anna Marias Lebens-, Krankheits- und Sterbegeschichte setzt auf Seite fünfundzwanzig des Familienbuches ein. Sie bildet eine eigenständige Geschichte im gesamten Text, indem sie leicht abgesetzt und mit einer eigenen Überschrift versehen ist: „1764 Lebens Laufff Unserer Herzallerliebsten Anna Maria, Deren Gebuhrt und Thauffen oben pag: 4: et: 5: zusehen“ 50 .

Den Anlaß zum Aufschreiben der Geschichte bildet der Tod des Kindes. Der Vater schreibt im Rückblick. Dabei beginnt Wirz seine Erzählung nicht mit dem Einsetzen der Krankheit, die zu Anna Marias Tod führte, sondern stellt ihr Leben von Geburt an im gesamten Ablauf dar, einschließlich früherer Krankheiten und gesunder Zeiten. Die Darstellung der gesunden Zeiten ist von Bedeutung, um den besonderen Charakter des Kindes hervorzuheben, aber auch, um im Kontrast zur Gesundheit die Intensität des Leidens während der Krankheit zur Darstellung zu bringen.

Zunächst erweist sich der Text auf der Ebene der dargestellten Praktiken als aussagekräftig. Wir erfahren etwa, daß Mayeli im Verlaufe ihrer Krankheit von fünf Heilpersonen behandelt wird. Drei davon werden vom Vater als Ärzte bezeichnet, zwei gehören dem weiteren Kreis von Heilpersonen an, eine Jungfer von S., deren Kur nicht beschrieben ist, und ein in Zürich ansässiger Mann, der eine Schröpfkur durchführt. Die Heilpersonen, vor allem die Ärzte, werden gewechselt, sobald sich ihre Kuren als nicht wirksam erweisen. Weitere Personen aus dem Verwandtschaftsnetz der Familie werden in Entscheidungs- und Betreuungsaufgaben eingebunden. Insgesamt wird durch diese Heilpersonen eine Vielzahl von Therapien durchgeführt. Die evakuierenden Therapien, Blattern-ziehen, Blutegel-ansetzen, Schröpfen und Laxieren werden alle mehrmals praktiziert. Die Arzneien zur inneren und äußeren Anwendung bleiben größtenteils vage bezeichnet: Neben Karottensaft werden Kräutertees, Tropfen, Pulver, Mixturen, Augen- und Kraftwasser verschrieben. Zweimal wird zur Unterstützung der Evakuierungstherapien eine Wasserkur mit Pfäferser Heilwasser verordnet, bei der das Kind jeweils 24 Flaschen Wasser trinken muß ${ }^{51}$. Hinzu kommen Mittel, die ausschließlich zur Linde-

50 Wirz, o. T. 25.

51 Werner Vogler, Heilendes Wasser. Uebersicht über die Geschichte des Pfäferser Bades. Separatdruck Terra plana (Mels 1985); Roman Schwizer, Leben und Vermächtnis des Balthasar Walthier, Badearzt in Pfäfers (1706-1756) (Zürich 1992). 
rung eingesetzt werden, zu denen auch die Verdunkelung des Krankenzimmers gehört. Die ärztliche Beobachtung des Kindes, vor allem gegen Ende der Geschichte, erfolgt mittels Urinschau in Ferndiagnose. Allmorgendlich bringt ein Bote den Urin des Kindes zum Arzt, der dann seine Schlüsse den Eltern mitteilen läßt.

Die Betrachtung der Behandlungsmethoden belegt die als Polypragmasie und Polypharmazie bezeichnete Überlagerung und Vielfalt der Mittel. Vor allem das mehrfache Schröpfen, Ziehen von Blasen und Ansetzen von Blutsaugern zeigt, daß im vorliegenden Fall das Bewußtsein von kinderspezifischen Behandlungsweisen, wie es sich in den Schriften des 18. Jahrhunderts abzeichnete, eher gering war. In Zedlers Universallexikon wurde festgestellt, daß zu den zarten Kinderleibern nur zarte Methoden paßten und insbesondere evakuierende Therapien den Kindern schädlich seien ${ }^{52}$. Auch war man der Meinung, daß der Urin von Kindern sich vom Erwachsenenurin unterscheide und sich daher nicht für Diagnosen eigne.

Deutlich wird jedoch, daß die Eltern - so die Darstellung des Vaters - alles unternehmen, immer neue Wege suchen, um das Kind zu retten und seine Leiden zu lindern.

Der Todestag des kleinen Bruders Hartmann markiert den Übergang von Anna Marias Krankengeschichte zum Sterbebericht. Doch von diesem Moment an bis zum Eintreten des Todes nimmt der Text weitere 17 Seiten in Anspruch. Es beginnt eine minutiöse Beschreibung der langsamen Abnahme der Kräfte, des Zerfallens und Verlöschens des kleinen kindlichen Körpers. Das Kind wird weiterhin medizinisch betreut, und täglich werden neue Prognosen gestellt. Gleichzeitig nimmt der Anteil der im Text eingefügten Gebete zu. Je stärker die physischen Kräfte des Kindes nachlassen, desto wichtiger werden die zwischen den Eltern und dem Kind inszenierten Gespräche. Mit den Passagen in direkter Rede verleiht der Vater dem Kind eine zunehmende Präsenz im Text, während er gleichzeitig seinen körperlichen Zerfall beschreibt. Den Tod des kleinen Hartmanns nehmen die Eltern zum Anlaß, erstmals mit Anna Maria über ihren Tod zu sprechen:

„als Wir Wieder Heimkamen, gienge seine L: Mutter zu Ihme in die Kammer, und sagte $\mathrm{Zu}$ Ihme, Nun ist dein L: Hartmeli Versorget und sein L. Seeleli im Himmel, bey seinem L. Heilland und dem L. Hänseli, Woltest du Jezt Lieber Gesund Werden, oder auch zu Ihme in den Himmel gehen, Es antworthete sehr anmuthig Ich wott Gern Gesund Werden Wen der Lieb Gott Wett, aber Doch Wetti Lieber in Himmel zu mim L: Heilland, und zu mein Lieb Hätmeli, und zu min Liebe Hänseli“ 53 .

Der Dialog als ein Stilmittel der Unmittelbarkeit und Authentizität ist hier der Ebene des Religiösen zugeordnet. Aus heutiger Perspektive liest sich das vom Va-

52 „Was nun die Cur der Kinder-Krankheiten anlanget, so muß man überhaupts ihr natürliche Schwachheit ja niemahls aus den Augen setzen, und daher solchs Medicamenta erwählen, welche sich zu solchen zarten Leibergen schicken." Vgl. Johann Heinrich Zedler, Grosses vollständiges Universal-Lexikon, Bd. 15 (Halle, Leipzig 1737) 648.

53 Wirz, o. T. 36-37. 
ter rapportierte Gespräch wie eine Strategie der Eltern, um das Kind mit dem Gedanken an den nicht mehr abzuwendenden Tod vertraut zu machen. Die Aussicht, in den Himmel zum Heiland zu gehen und dort die zwei verstorbenen Brüder wiederzusehen, führen Mayeli dazu, den Tod einer Genesung vorzuziehen. Doch in der Art und Weise, wie der Vater Anna Marias Antwort präsentiert, läßt er sie keineswegs als naiv, sondern als seelisch reif und von großer religiöser Stärke erscheinen. Es scheint, sie habe ihre Entscheidung mit Bedacht und im Vertrauen auf Gott gefaßt.

„als Es mich hörte Seüfzen, Daß doch der Liebste Heilland bald die L. Hlgen. Engeli senden Wolle, sein Liebes Seeleli abzuholen, Waren Wieder seine Ersten Worth jJa! Und als Es bald daruf Von seiner L: Mutter gefraget Wurde, Kennest Du mich, antworthete Es Wiederum mit dem aller Liebereichsten, und sanftesten Thon und Stimme Jja, Wer bin ich Dann Mi Liebs Mötterli, Wird dein L: Heylland bald kommen Jja! Siehest Du ihn und die L: Engeli jJa, Wilt Du gerne Zu Ihnen, und deinen L: 2: brüederlenen in den Himmel Ach jJa, Ich und seine Geschwüsterte fragten Es auch, und die Frau Baas, ob es uns kenne Es antworthete allen, mit gleicher Liebe und Sanft Muth sein anmutiges jJa!“54

Diese eindrückliche Passage handelt vom Bestätigen und Herstellen von Beziehungen und dem Übergang des Kindes aus dem weltlich-familiären in ein himmlisches Beziehungsnetz. Das weltlich-familiäre Beziehungsnetz wird durch die Eltern, die Geschwister und die Baas vertreten, das himmlische durch den Heiland, die Engel und die zwei verstorbenen Brüder. Mit der Frage „Kennst du mich, deine Mutter, deinen Vater, deinen Bruder etc.?“ werden die noch bestehenden Beziehungen bestätigt, mit der Frage „Willst du zum Heiland, zu den Engeln, zu deinen Brüdern gehen?" wird das Kind in sein neues Beziehungsnetz eingeführt. Auf alle ihm gestellten Fragen antwortet das Kind mit dem gleichen sanften „Ja“. Das Kind, so die Mitteilung dieser Textstelle, ist bei vollem Bewußtsein und freut sich auf seinen Tod. Allerdings beinhaltet die Passage auch die Aussage, daß die Welt, in die das Kind bald eintreten wird, ihm nicht unbekannt, die religiöse Erziehung, die es erhalten hat, gelungen ist. Mayeli kennt seinen Heiland und die Engel und erkennt sie daher auch.

Bis auf Seite 49 hat Wirz sein Familienbuch paginiert. Als die lange Erzählung sich ihrem Ende zuneigt, durchbricht der Vater diese Ordnung und unterläßt die Seitennumerierung. Das nahende Ende bildet sich durch eine vermutlich unbewußte Unterlassung auf der Ebene der formalen Ordnungsstruktur des Textes ab.

Das Sterben des Kindes wird folgendermaßen beschrieben:

„[Am] Abend Da Waren seine 2 Letzten Worte Gott Sohn Wasch mich mit Dine Blot, und ach Jesu du min ayns: darmit Wurde es Sprachloß, und konnte Wegen Vielem Schleim im Mündli nichts mehr Schlucken, der Husten wurde Schwächer und das Röcheln hefftiger, gegen die Nacht ohngefahr Um 8: Uhren brachen die Gichten Wieder an Jhme auß, daß es beständig mit dem Linken Händli und Beindli zuckte und Wann es solche aufgehoben Erbärmlich gezittert und Daß Wehrete Bis

54 Ebd. 44-45. 
gegen 3: Uhren des Morgens, Werend Diesen Gichteren auf der Linken Seithen Nahme Es Daß Rechte Händli hinauf gegen dem gesichtli, Winkte mit dem Zeigefingerli und dem Ganzen Händli Von ohngefahr 9: Uhren bis nach Mitternacht, beständig forth, So artlich, Wie Es in Seinem Leben gewunken, Wann es Jemand herbey Locken Wollen, sonder allen Zweiffel Lockte Es seinen L: Herren Jesum, oder seinen Schuzgeist Herbey, Die Gichter Dauerten bis 3: Uhren Samstag Morgens... Doch Von Da an Nahme Es ab, nach und nach Wie ein Liechtli, das L: Näßli, Hand und füeßli Wurden kalt, der Puls und Odem Je Länger Je Schwächer, der < kalte $>$ Schweiß Lage Ihme auf der Stirne, den wir Fleißig abtrockneten, Bis Ein Viertel nach 7: Uhren Nahme Ich Wieder Ein Buch und Bättete, Wenige Minuten Darauf Ruffte die <L:> Mutter Jezt! Ich Sahe nach Ihme Es hollete 2: oder 3: Mahl Athem, und Wir meinten es seye gethan, nach Verfluß aber 1: oder 2: Minuthen Rümpfte Es Einmahl sanfft <sein L: Mündli> und das 2te Mahl zogen Ihme die Gichter solches hart midsich gegen der Rechten Seithe zu, und darmit Verschied es ohne Ein fingerli zuverzühenen". 55

Erzählt werden in diesem Sterbebericht des Vaters über den Tod seines Kindes zwei Geschichten: die Geschichte des verlöschenden Kinderkörpers und die Geschichte eines Abschieds von dieser Welt und gleichzeitiger Ankunft in der anderen Welt, die Geschichte des Körpers und jene der Seele im Tod. Geprägt ist die Passage von der minutiösen Beobachtung des sterbenden Körpers und seiner Äußerungen und dem Versagen der körperlichen Funktionen, vom Verlust der Sprache über das Erkalten der Gliedmassen und Nachlassen von Puls und Atem bis hin zum letzten Zucken des Mundes. Exakt beschreibt der Vater, wie das Kind in krampfartigen Anfällen zuckt, zittert und sich verrenkt. Mitten in der Beschreibung dieses harten Kampfes plaziert der Vater die andere Geschichte. Das Kind winkt, wie es auch in seinem Leben gewinkt hat, und lockt mit seinem Winken Jesus oder seinen Schutzgeist herbei, der es abholen soll. In der väterlichen Deutung der Körperverrenkungen seines sterbenden Kindes wird das, was soeben noch „erbärmliches Zittern“ war, zu einem „artlichen Winken“. Doch das anmutige Bild des friedlich-winkenden Kindes bleibt nur für einen kurzen Moment stehen. Die kurze Passage genügt dennoch, um die im körperlichen Zerfall enthaltene Brutalität des Sterbens zu relativieren und das Kind in der Erinnerung sanft und zufrieden sterben zu lassen.

Eine Lektüre von Anna Marias Krankengeschichte, die das Augenmerk ausschließlich auf die körper- und medizingeschichtlichen Fragen im engeren Sinn richtet, würde die Qualität des Textes nicht erfassen können. Erst unter Einbezug der narrativen Gestaltungsmittel wird die Bedeutungsdichte des Textes greifbar. Zum einen beschreibt der Vater den langsamen aber steten körperlichen Zerfall seiner Tochter. Durch die Dialoge zwischen dem Kind und den Eltern, die der Vater im Text in stetig zunehmender Dichte einbaut, wird das Kind andererseits immer gegenwärtiger und lebendiger. Der Vater präsentiert Sterben und Tod als religiös vermittelten Willensakt des Kindes. Die zwei gegenläufigen Aussagebewe-

55 Ebd. o. S. 
gungen des Textes umfassen Medizin und Religion als zwei Deutungs- und Handlungsmuster, die im Kontext von Krankheit nebeneinander zum Tragen kommen und gleichermaßen relevant sind.

So berührend das Familienbuch von Hartmann Wirz in vielen Passagen zweifellos ist, kann es nicht darum gehen, die Gefühle, die hinter diesen Berichten stehen, zu eruieren. Auf den Grad der Emotionalität von Eltern gegenüber ihren Kindern kann weder aus der Kürze von Todesnachrichten - wie dies oft geschah - noch aus der Länge oder Intensität eines Textes geschlossen werden. Bereits die stark verkürzte Lektüre dürfte aber gezeigt haben, daß und auf welche Weise der Vater Hartmann Wirz in seinem Text ein elterliches Selbstbild präsentiert, in dem das liebende Umsorgen und Pflegen von Kindern durch Väter, Mütter und andere Personen Teil des familiären Alltags darstellte.

Abschließend einige zusammenfassende Überlegungen zu den zwei Texten vor dem Hintergrund der fünfzig insgesamt untersuchten Texte:

Die beiden kurz vorgestellten Texte sind unediert, ebenso wie die rund fünfzig Texte, die den Ausgangspunkt einer umfassenderen Untersuchung darstellten. Die Wahl von unediertem Material hat Gründe. Ein Ansatz, der sich nicht primär für die Biographien der Autorinnen und Autoren und auch nicht ausschließlich für die beschriebenen Inhalte, sondern für das Selbstzeugnisschreiben selbst als Praxis interessiert, führt vorzugsweise über die Konzentration auf unediertes Material. Oft beurteilte die Forschung Mischformen, fragmentarische und untypische Texte als zur Analyse ungenügend. Es wurden Anforderungen nach Narrativität oder Reflexion gestellt, die zum Ausschluß von 95\% aller Selbstzeugnisse führten ${ }^{56}$. Um sich dem Selbstzeugnisschreiben in seiner historischen Bedeutung nähern zu können, müssen aber - ganz im Gegenteil - auch die vielen spröden, fragmentarischen, „nur" berichtenden oder gar völlig formalisierten Texte konsequent untersucht werden. Es gilt, die autobiographischen Textsorten in ihrer Vielfalt wahrzunehmen, ohne sich an engen Gattungsbegriffen von „Autobiographie“ oder „Tagebuch“ zu orientieren. An unediertem Material zeigen sich die Merkmale der Normalität - Uneinheitlichkeit, abrupte Abbrüche, unfertige Sätze, Unleserlichkeiten u. a. - auf schonungslose Weise. Die methodische Entscheidung für unedierte Texte führt deshalb zum Ansatz, Selbstzeugnisse nicht mehr als Textsorte mit formal bestimmbaren Kriterien aufzufassen, sondern das Selbstzeugnisschreiben als eine kulturelle Praxis zu verstehen. Diese bislang weniger untersuchten Texte sind es, die den Blick auf die Praxis des Selbstzeugnisschreibens in seiner Normalität eröffnen.

Die Arbeit mit dieser „Normalität“ des Selbstzeugnisschreibens bewegt sich im ständigen Spannungsfeld von typischen und untypischen, unspektakulären und spektakulären Quellen, von Normal- und Ausnahmefall des Selbstzeugnisschreibens. Hierzu schrieb Roy Porter im Rahmen eines Überblicksartikels zur Körper-

56 Z. B. bei Peter Alheit, Morten Brandt, Hans-Rüdiger Müller, Frank Schömer, Konfigurationen der Bildung. Drei Fallstudien zur Leibthematik im autobiographischen Text um 1800 (Göttingen 2001). 
geschichte: „The first-hand written record... is largely silent - and where it is eloquent, it is probably unrepresentative. " 57 Die Texte, die sich - wie jener Eschers oder Wirz' - zu ausführlichen Analysen eignen, sind dabei zumeist die außerordentlichen, gesprächigen und damit nicht repräsentativen. Wie sie zustande kommen - warum also gerade Hartmann Wirz 36 Seiten über seine kranke und sterbende Tochter verfaßte, während andere Autorinnen und Autoren den Tod von Kindern auf einer Zeile notieren -, läßt sich kaum erklären. Doch ermöglichen vor allem solche außergewöhnlichen Texte die Darstellung von Wahrnehmungs-, Deutungs- und Handlungsmustern sowie die Analyse der narrativen Strategien des Schreibens. Der zusätzliche Einbezug normaler, unspektakulärer Texte dient dann vor allem der Einordnung und dem Rückbezug dieser Texte in den Rahmen des Selbstzeugnisschreibens.

Einen weiteren Hintergrund für die Interpretation der Selbstzeugnisse bilden die verschiedenen Diskurse, die sich im 18. Jahrhundert und verstärkt ab 1750 rund um den Körper entfalteten. Die in den Texten beschriebenen Erlebnisse und Erfahrungen verweisen zurück auf die diskursiven Bedingungen, die diese formten. Sei es in Krankheitsbeschreibungen, beim Schreiben über Gesundheit, Tod, Ernährung, Geschlechterbeziehungen, Erziehung, Hygiene, Tugend oder Onanie, immer wieder läßt sich zeigen, wie das Schreiben über den Körper sprachlich codiert und an zeitspezifische Diskurse gebunden ist ${ }^{58}$. Allerdings können Selbstzeugnisse nicht als bloße Reaktionen auf Diskurse gelesen werden. Als Angehörige der gebildeten Schicht sind Autorinnen und Autoren von Selbstzeugnissen wie etwa Johann Caspar Escher - gleichermaßen Rezipienten wie Produzenten öffentlicher Diskurse. Im Verfassen von Selbstzeugnissen produzieren sie die Diskurse selbst mit, deuten sie um, definieren sie neu und eigenständig. Dies ermöglicht eine Sicht, in der Diskurse nicht als abstrakte Größen verstanden werden, sondern in Zusammenhänge der Praxis eingebettet sind ${ }^{59}$. Denn Selbstzeugnisse reflektieren die Auseinandersetzung mit Normen und Diskursen, aber auch mit sozialer Praxis und Lebenswelt.

Körpergeschichte auf der Basis von Selbstzeugnissen erweist sich somit immer auch als Diskursgeschichte. Dennoch ermöglichen die Analysen Einblick in die subjektiven und eigenwilligen Artikulationsweisen und Wahrnehmungen von Leiblichkeit, die sich in den Selbstzeugnissen auf mehrfache Weise artikulieren, dies etwa als individuelle Auswahl aus konkurrierenden Wissensmodellen oder als subjektive Deutungen im Sinne der eigenen Aussageabsicht.

57 Roy Porter, History of the Body, in: Peter Burke (Hrsg.), New Perspectives on Historical Writing (Oxford 1991) 206-232, 210.

58 Vgl. Jakob Tanner, Wie machen Menschen Erfahrungen? Zur Historizität und Semiotik des Körpers, in: Bielefelder Graduiertenkolleg (Hrsg.), Körper Macht Geschichte. Körpergeschichte als Sozialgeschichte (Bielefeld, Rieden 1999) 16-34.

59 Vgl. auch Katbleen Canning, Problematische Dichotomien. Erfahrung zwischen Narrativität und Materialität, in: Historische Anthropologie 10 (2002) 163-182, 167. 